\title{
Estudio de la gestión de las estrategias de riego en los olivares tradicionales.
}

\author{
Molina-Moral, J.C. ${ }^{1}$, Moriana Elvira, A ${ }^{2}$, Pérez-Latorre, F.J. ${ }^{1}$
}

Dpto. Área Mecánica de Fluidos, Campus Científico Tecnológico Linares. Universidad de Jaén.; jcmm001@red,ujaen.es; fjperez@ujaen.es

2 Dpto. Agronomía ETSIA, Universidad de Sevilla; ammoriana@us.es

Resumen: El riego en el olivar tradicional permite aumentar el rendimiento, pero supone un coste adicional que debe optimizarse. La provincia de Jaén (España) es el lugar del mundo donde el riego de este tipo de olivar es más importante. La gran homogeneidad existente en los sistemas de riego localizados y la tipología de las instalaciones y equipos instalados en estos cultivos tradicionales en esta provincia, permite estudiar y caracterizar diferentes estrategias para el diseño de proyectos de riego que no afecten al rendimiento del cultivo, cuando se disminuye la dosis de aplicación.

El objetivo de este trabajo es caracterizar el riego del olivar en una amplia región. Para ello, se estudia la variación del contenido de agua en el suelo de una finca típica de olivos bajo diferentes escenarios de precipitación, demanda evaporativa, tasa de aplicación y disponibilidad temporal de agua de riego para estimar las necesidades y estrategias de riego más adecuadas.

El estudio se ha realizado con los datos disponibles de 18 estaciones agroclimáticas con las que se han establecido patrones de precipitación máxima, media y mínima.

Se consideraron tasas de aplicación diaria en instalaciones típicas de 3,2 (1 sector), 1,6 (2 sectores) y 1,1 (3 sectores) mm día-1, combinadas con tres tipos de disponibilidad de agua: diaria, sólo durante 20 días al mes y con ausencia total en agosto. Los resultados se analizaron desde el punto de vista del momento de inicio del estrés hídrico, las necesidades de riego, las horas de funcionamiento del sistema y la dosis de riego.

Los resultados sugieren que la estrategia de riego de 2 sectores con ausencia de riego en agosto podría ser económicamente la de mayor interés para el olivicultor. Por otro lado, se obtiene que la estrategia que garantiza un mejor perfil hídrico en el suelo es la que corresponde a la implantación de la sectorización en un solo sector y la aplicación de la estrategia de riego E3: RDI, calendario de marzo a septiembre con riego deficitario controlado en agosto.

Palabras clave: cambio climático, endurecimiento del hueso, estrés hídrico, disponibilidad de agua. 


\title{
XXXVIII Congreso Nacional de Riegos CARTAGENA 2021
}

\section{Study of the management of irrigation strategies in traditional}

\section{olive groves.}

\author{
Molina-Moral, J.C. ${ }^{1}$, Moriana Elvira, A², Pérez-Latorre, F.J. ${ }^{1}$
}

Dpto. Área Mecánica de Fluidos, Campus Científico Tecnológico Linares. Universidad de Jaén.; jcmm001@red,ujaen.es fjperez@ujaen.es;

2 Dpto. Agronomía ETSIA, Universidad de Sevilla; amoriana@us.es

\begin{abstract}
Irrigation in traditional olive groves increases yields, but entails an additional cost that must be optimised. The province of Jaén (Spain) is the place in the world where irrigation of this type of olive grove is most important. The great homogeneity existing in the localised irrigation systems and the typology of the installations and equipment installed in these traditional crops in this province, allows the study and characterisation of different strategies for the design of irrigation projects that do not affect the crop yield when the application dose is reduced.
\end{abstract}

The aim of this work is to characterise olive orchard irrigation in a wide region. For this purpose, the variation of water content in the soil of a typical olive groove farm under different scenarios of precipitation, evaporative demand, application rate and temporal availability of irrigation water is studied in order to estimate the most appropriate irrigation needs and strategies.

The study has been carried out using data available from 18 agro-climatic stations with which maximum, average and minimum rainfall patterns have been established.

Daily application rates in typical installations of 3.2 ( 1 sector), 1.6 ( 2 sectors) and 1.1 ( 3 sectors) mm day1 were considered, combined with three types of water availability: daily, only for 20 days per month and with total absence in August. The results were analysed in terms of time of onset of water stress, irrigation requirements, hours of system operation and irrigation dose.

The results suggest that the 2-sector irrigation strategy with no irrigation in August could be economically the most interesting for the olive grower. On the other hand, it is obtained that the strategy that guarantees a better water profile in the soil is the one that corresponds to the implementation of sectorization in a single sector and the application of the E3 irrigation strategy: RDI, from March to September calendar with controlled deficit irrigation in August.

Key words: climate change, stone hardening, water stress, water availability. 


\section{Congreso Nacional de Riegos CARTAGENA 2021}

\section{Introducción}

A partir de los años 90 del siglo pasado, se produjo un aumento de la superficie de olivar en todo el mundo; la mayoría de estas nuevas superficies eran de regadío y más densos [1]. Sin embargo, las explotaciones olivareras tradicionales siguen siendo las más comunes. Además, estas plantaciones tradicionales de baja densidad son los más importantes en las zonas agrícolas áridas, comúnmente asociadas a la baja disponibilidad de agua. España es el primer productor mundial de aceite de oliva y es el país con mayor superficie [1], lo que supone una gran diversidad de sistemas de cultivo. Jaén es la provincia española que mayor superficie dedica a esta especie, con 482.427 hectáreas, de las cuales 243.888 hectáreas son de regadío, [2] (MAPA, 2018). Al contrario de lo que ocurre en otras partes del mundo, la mayor parte de la superficie de regadío en esta zona es de olivar tradicional de baja densidad (88\% de los olivares de regadío frente al 12\% de los intensivos [3].

La sostenibilidad del agua está relacionada con dos condiciones principales. Por un lado, la conservación de los recursos hídricos en calidad y cantidad a largo plazo [4] y por otro aquellas políticas que supongan la fijación de la población en las zonas rurales. De este modo, las gestiones agrícolas sostenibles se aplicarán cuando se obtengan suficientes ingresos económicos de la explotación [5]. El riego de las plantaciones tradicionales es un muy buen ejemplo. El aumento esperado del rendimiento podría mejorar la gestión sostenible en los olivos tradicionales de secano, que suele estar limitada por los bajos beneficios. Por otro lado, las previsiones de cambio climático auguran una menor pluviometría y una mayor variabilidad que puede afectar a las etapas fenológicas más sensibles del cultivo como son la floración y la maduración [6]. Entonces, las necesidades hídricas aumentarían y las plantaciones tradicionales de olivos de secano, que hoy en día tienen un rendimiento aceptable, podrían reducir fuertemente sus beneficios y se abandonarían. Los que suponen que los agricultores consideran el riego como la solución a largo plazo a los problemas de bajos ingresos y toda la sociedad podría considerar como herramienta para aumentar la sostenibilidad de estos sistemas agrícolas.

El olivo es un cultivo frutal tradicional de secano, uno de los más resistentes a las condiciones de estrés hídrico. Las funciones de producción sugieren una alta productividad del agua de riego con un gran incremento del rendimiento, con un bajo aumento de la evapotranspiración del cultivo [7]. Sin embargo, los trabajos en olivares de baja densidad no han presentado esta clara respuesta [8]. Los olivares tradicionales de baja densidad se sitúan en zonas con poca cantidad de recursos hídricos. Por tanto, aumentar la superficie de riego de un sistema agrícola no puede ser una estrategia sostenible. Además de la escasez de agua, estas zonas suelen tener problemas de disponibilidad de agua a lo largo de la temporada (con una temporada de riego corta o un tiempo de riego corto). Esto supone un problema adicional en la programación y diseño de los sistemas de riego. Aunque el olivo es una especie resistente a la sequía, no todos los estados fenológicos a lo largo de la estación son igual de resistentes e incluso una combinación en la duración y el nivel de estrés hídrico podría ser importante [9]. Los periodos de floración, cuajado y acumulación de aceite son los más sensibles a las condiciones de estrés hídrico, [10]. Por otro lado, niveles relativamente severos de estrés hídrico durante la fase de endurecimiento del hueso no reducen el rendimiento final [7]. Por lo tanto, se podría considerar el riego deficitario, estimando el momento en el que se van a realizar estas condiciones de estrés hídrico. Por otro lado, los olivares tradicionales, aun contando con este aumento de rendimiento, tendrían una capacidad de inversión en riego muy reducida, puesto que, los sistemas de riego actuales tienen limitaciones en la capacidad de bombeo y en las dosis de agua aplicada. Esto podría ser una limitación importante en la programación del riego.

El objetivo de este trabajo es estimar la reserva hídrica del suelo en las condiciones de una plantación tradicional de baja densidad de olivos considerando varias limitaciones a nivel de finca y posibles 


\section{Congreso Nacional de Riegos CARTAGENA 2021}

escenarios en el comportamiento de las lluvias. De esta forma, los resultados aportarán una visión holística de la sostenibilidad del riego en este sistema agrícola. Se han considerado las condiciones climáticas de la provincia de Jaén (España) por ser la zona de olivar tradicional más importante del mundo. Con el fin de incluir las limitaciones más comunes para la programación del riego, además de la cantidad de agua, se han tenido en cuenta el tiempo de riego disponible a lo largo de la temporada y la organización del sistema de riego.

\section{Materiales y métodos (Trabajo de innovación: descripción de la innovación)}

\section{1. Área de estudio.}

La zona de estudio comprende la provincia de Jaén (España), que tiene una superficie de 13.489 $\mathrm{km} 2$, en la que el olivar es el cultivo frutal más importante de la superficie (582.427 hectáreas), de las cuales 249.888 hectáreas son de regadío (alrededor del $43 \%$ ). Esta zona es la mayor productora mundial de aceitunas y aceite con más de 60 millones de olivos, y una producción media total en la última década de 2.214.021 toneladas de aceitunas, y 480.025 toneladas de aceite de oliva, [2], lo que supone que este cultivo es el primer y principal motor a nivel socioeconómico en la provincia.

\subsection{Características de la instalación de riego.}

En la actualidad, se presentan varios sistemas en las zonas olivareras. A partir de los años 90 del siglo pasado, el aumento de la densidad en los olivares ha ido en aumento y se ha cambiado parte de los tradicionales, de secano y baja densidad, por los de regadío de muy alta densidad, [5]. Sin embargo, las plantaciones de baja densidad siguen siendo los más importantes en superficie, así el olivar tradicional, con marcos de plantación de 9 a 12 metros y densidades entre 80-120 plantas/ha [12]. Según el Plan Director del Olivar, [13], la densidad media de plantación en la provincia de Jaén es de 117 plantas/ha. Algunas de estas explotaciones tradicionales han pasado de ser de secano a ser de regadío. El sistema de riego más habitual es el de dos goteros por árbol, lo que permite una dosis de $16 \mathrm{~L} \mathrm{h-1}$ por planta [14-15]. Por otro lado, la disponibilidad de agua es muy limitante y comúnmente, existen serias restricciones en cantidad y tiempo de riego [16]. Para maximizar la superficie regada, se divide la cantidad máxima de riego diario entre 2 o 3 sectores, para aumentar la superficie regada [17]. Así pues, la cantidad de agua disponible se divide entre 1, 2 o 3 partes para regar la plantación completa (por lo que se denomina a partir de aquí 1,2 o 3 sectores). En cuanto al tipo de sectorización utilizado, Peragón y otros [18], realizaron una encuesta en las comunidades de regantes de la provincia, mostrando que más del $90 \%$ de las instalaciones tienen una sectorización en 3 sectores.

De esta forma, la parcela agrícola tipo considerada está formada por olivares maduros con una distancia común entre árboles de $10 \times 10$ m, lo que nos da una densidad de 100 olivos por hectárea, con un sistema de riego por goteo que consiste básicamente en una elevación desde una captación de agua hasta un depósito de agua y posterior rebombeo a la explotación olivarera, con la instalación de una red de tuberías primarias, secundarias, terciarias y de goteo que terminan en dos goteros autocompensantes por árbol de 8 litros/hora de caudal instantáneo $(0,44$ 1/ha). Con el sistema de riego propuesto como caso de estudio, la programación del riego se limitó en función de un número máximo de horas disponibles para el riego (20 horas día-1). Así, la dosis máxima a aplicar depende de la realización de una determinada sectorización en 1, 2 ó 3 sectores y permite utilizar el agua disponible el 100\%, 50\% y $33 \%$ del tiempo. Esto supone limitar la capacidad de riego de los olivares a 3,2 mm día-1 (1 sector), 1,6 mm día-1 (2 sectores) y 1,1 mm día-1 (3 sectores). Para cada una de estas estrategias se caracterizó mensualmente la reserva disponible en el suelo. En las programaciones de riego se consideró que al 


\section{Congreso Nacional de Riegos CARTAGENA 2021}

inicio del año agrícola (octubre) se utilizaba una reserva de $10 \mathrm{~mm}$ (cantidad muy cercana a la realidad en condiciones naturales en la zona estudiada). Se consideró que la eficiencia de aplicación del sistema de riego utilizado, correspondiente a un sistema de riego por goteo localizado, era del 95\%.

\subsection{Variables climáticas.}

Los datos climatológicos se realizaron a través de la Red de Información Agroclimática de Andalucía, [19], constituida por estaciones meteorológicas automáticas estratégicamente ubicadas. A partir de ellos se calculó los datos de los años agrícolas (octubre-septiembre) durante el periodo 2001-2020. En total, se han considerado 18 estaciones agroclimáticas activas distribuidas en 7 regiones agrícolas. Los resultados de evapotranspiración de referencia (ETo) utilizados son los calculados por la Red de Información Agroclimática de Andalucía (RIAA), que la determina utilizando los datos climáticos estándar recogidos en el modelo matemático Penman-Montheith, según establece la FAO [20]). La precipitación efectiva se estimó como aquella que produce el 75\% de la infiltración del agua de lluvia en el suelo [21]).

El análisis de las variables climáticas correspondientes a la precipitación y evapotranspiración consistió en realizar un estudio de variabilidad de los valores extremos (máximos y mínimos) y de los valores medios [22], determinando los valores medios mensuales y el total anual para cada una de las estaciones agroclimáticas consideradas (Figura 1). Este procedimiento permite clasificar las estaciones agroclimáticas considerando sólo aquellas que presentan valores similares, por encima o por debajo de los resultados obtenidos. Para acercarse a la realidad, se ha calculado también el valor de la mediana para eliminar el sesgo de los valores extremos.

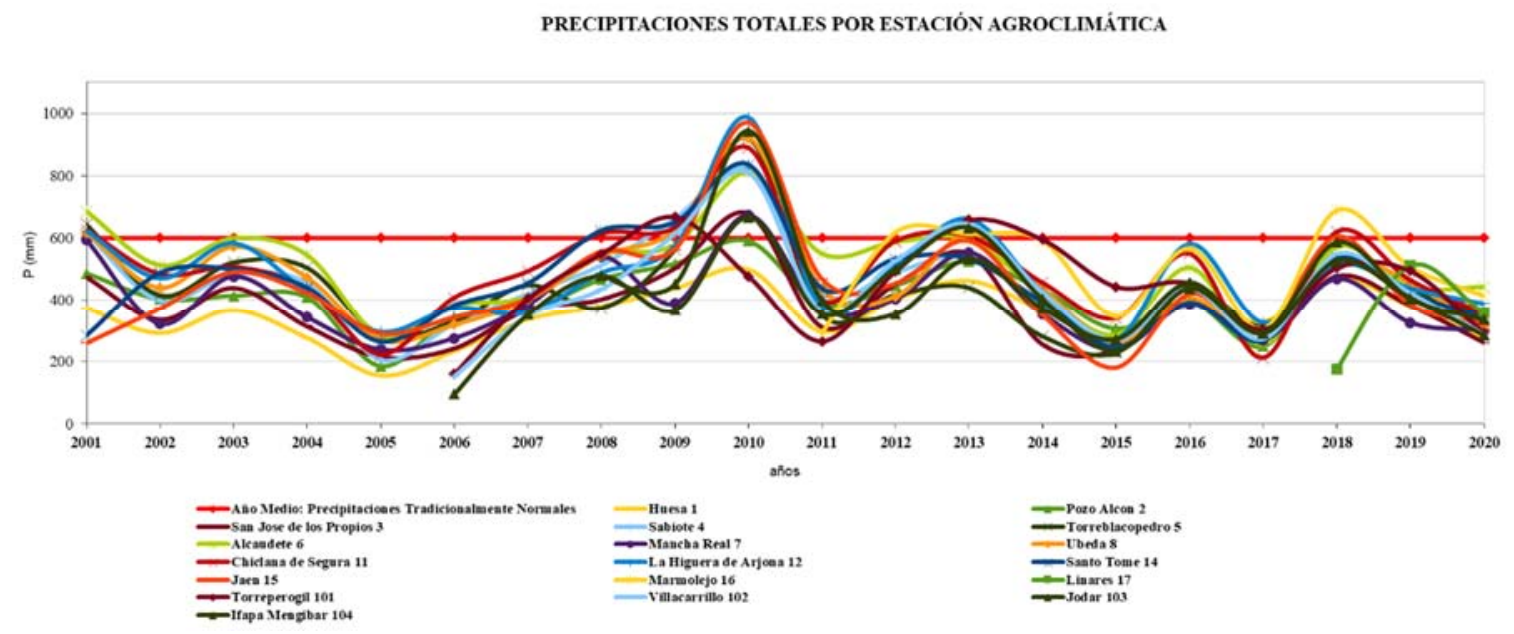

Figura 1: Precipitación anual por estación agroclimática.

De este modo, se clasificaron las estaciones agroclimáticas en relación con la variable de la precipitación según los resultados, y se consideraron tres escenarios basados en los datos reales de la precipitación en toda la serie histórica:

- Escenario 1; Cuando la precipitación media mensual es superior a la media, se elige como dato la mediana de la precipitación máxima, considerando como años de mayor precipitación aquellos que superan los $581 \mathrm{~mm}$.

- Escenario 2; La media de las precipitaciones mensuales. 


\section{Congreso Nacional de Riegos CARTAGENA 2021}

- Escenario 3; Cuando la precipitación media mensual está por debajo de la media, se elige como dato la mediana de la precipitación mínima, considerando como años de menor precipitación los que están por debajo de $292 \mathrm{~mm}$.

\subsection{Constantes de referencia: tipo de suelo, profundidad de las raíces, cobertura del dosel y grado de desarrollo} del cultivo.

La determinación de las necesidades de riego y el momento de entrada en estrés hídrico se establecieron siguiendo el método del balance hídrico, de forma que se considera que el agua consumida es la suma del agua de riego y de las precipitaciones, [23]). En este balance, el agua útil o disponible en el suelo para la planta estará entre los límites superior e inferior.

En el caso del estudio, el tipo de suelo elegido fue franco-arcilloso (por ser el más común en la zona), con las siguientes características: punto de marchitez (PT) $(\mathrm{cm} 3 / \mathrm{cm} 3)$ 0,17; capacidad de campo $(\mathrm{CF})(\mathrm{cm} 3 / \mathrm{cm} 3)$ 0,36; densidad aparente del suelo $(\mathrm{cm} 3 / \mathrm{cm} 3)$ 1,33 [24]. La humedad disponible para la planta se estimó utilizando un nivel de agotamiento permisible (ADL) del 75\%, que es el nivel usualmente recomendado [25]. Se estableció una profundidad radicular útil de $1,0 \mathrm{~m}$. según diferentes trabajos [7-9]. La evapotranspiración de los cultivos (ETc) se estimó según la metodología propuesta por Allen y otros [26]

- $\quad \mathrm{ETc}=\mathrm{ETo}^{*} \mathrm{Kc}{ }^{*} \mathrm{Kr}$. [1]. Dónde [1]: ETo: Evapotranspiración del cultivo de referencia.

- Kc: Coeficiente adimensional del cultivo.

- Kr: Coeficiente adimensional de reducción de la evaporación.

Los valores de Kc utilizados (Tabla 1) son los sugeridos por Pastor y otros, [15]. En el caso de estudio, se ha considerado que el valor del coeficiente de reducción $\mathrm{Kr}$ tomará un valor de 1, ya que el cultivo sombreará más del 50-60\% de la superficie del suelo, según Castel y Fereres, [27]). En cuanto a la fenología del cultivo en la zona, la fecha media de plena floración tuvo lugar en mayo), y el inicio del endurecimiento del hueso está fechado en julio, [26].

Tabla 1: Variación mensual del coeficiente Kc.

\begin{tabular}{|l|l|l|l|l|l|l|l|l|l|l|l|l|}
\cline { 2 - 11 } \multicolumn{1}{c|}{} & Ene. & Feb. & Mar. & Abr. & May. & Jun. & Jul. & Ago. & Sep. & Oct. & Nov. & Dic. \\
\hline Kc & 0,5 & 0,5 & 0,65 & 0,65 & 0,65 & 0,6 & 0,6 & 0,6 & 0,6 & 0,65 & 0,65 & 0,5 \\
\hline
\end{tabular}

\subsection{Programa de cálculo.}

Para el cálculo de la programación y dosis de riego se utiliza la metodología del balance hídrico, tal y como se recoge en el no 56 de la FAO [26]). El programa utilizado es una adaptación del programa de cálculo y determinación de las "Necesidades Hídricas del Olivar" del IFAPA, ([19], de acceso abierto, previo registro, y publicación online en Internet desde 2015 (https://www.juntadeandalucia.es/agriculturaypesca/ifapa/servifapa/recomendador-olivar), en el que se introdujeron las variables mencionadas, con los datos medios de la estación más lluviosa y más seca del total de 18 estaciones agroclimáticas.

\subsection{Estrategias de riego.}

Los escenarios anteriores se caracterizaron además en función de la disponibilidad de agua, seleccionando los más comunes en la zona de estudio: 


\section{Congreso Nacional de Riegos CARTAGENA 2021}

- Estrategia 1 (IDD): Riego todos los días del mes, [14]). Esta simulación pretende reflejar aquellas instalaciones de riego sin limitaciones de tiempo ni de disponibilidad de agua durante el periodo de riego (marzo - septiembre), donde el uso del agua es de máxima eficiencia todo el tiempo.

- Estrategia 2 (ID20): Riego 20 días al mes. Se trata de instalaciones con limitaciones temporales o de disponibilidad de agua (aproximadamente el 30\%). Esta estrategia es similar a la anterior, pero dejando los fines de semana libres, similar a un calendario laboral convencional, [29]).

- Estrategia 3 (RDI): Riego deficitario regulado. Estas instalaciones no disponen de agua durante un periodo de tiempo determinado que coincide con el periodo de parada estival del olivar. Existen diferentes estudios de estrategias de riego deficitario en diferentes cultivos [30]), así como en olivar en los que se proponen importantes recortes de riego, tanto en olivar tradicional [31]) como en olivar intensivo o superintensivo ([7-32]. En la zona de estudio, en la práctica, estos cortes se establecen en el periodo comprendido entre julio y agosto. La aplicación del riego en condiciones de estrés hídrico moderado produce un aumento tanto de la calidad del aceite de oliva como de la tasa de acumulación, [33-34]En el caso particular de este estudio, el mes de agosto se ha establecido sin riego.

\section{Resultados}

\subsection{Precipitación}

Los años medios de todas las estaciones presentaron un patrón de precipitaciones muy similar, con un periodo húmedo entre septiembre y mayo, con máximos en noviembre y marzo, y un periodo muy seco durante el final de la primavera y el principio del verano. También se han obtenido los valores extremos de precipitación y sus tendencias, en los que se observa que, en general, el rango entre los valores extremos de precipitación es mayor en los años húmedos que en los secos, la figura 2 muestra la variabilidad de los datos a lo largo de los años medios (a) y los valores máximos y mínimos encontrados.

En la figura 2, se destacan varios hechos. En primer lugar, las precipitaciones tienen una distribución variable tanto en los años lluviosos como en los secos. En segundo lugar, el intervalo o rango entre las precipitaciones máximas y mínimas suele ser mayor en los años lluviosos que en los secos. En tercer lugar, la mayor parte de las precipitaciones máximas se sitúan por debajo de los $600 \mathrm{~mm}$, mientras que para las mínimas tenemos que éstas en la mayoría de las ocasiones no llegan a los $300 \mathrm{~mm}$, aunque muestran una tendencia a acercarse significativamente a partir de 2016. Los datos de estas 18 estaciones agroclimáticas se utilizaron para obtener los escenarios de precipitación, media y mínima.

MAXIMAS Y MINIMAS PRECIPITACIONES CON TENDENCLAS

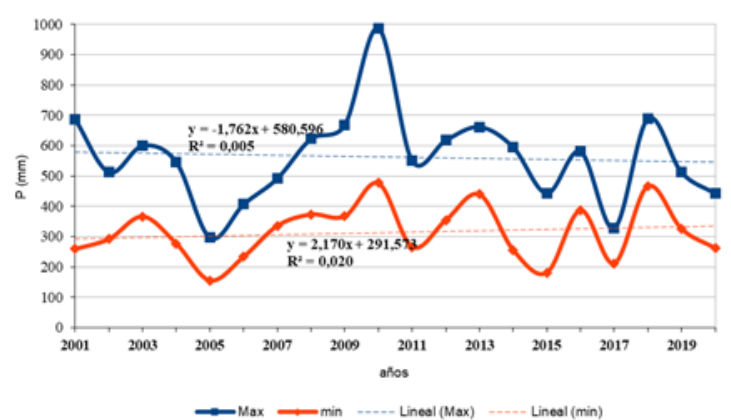

Figura 2: Precipitaciones máximas y mínimas y tendencias. 


\subsection{Evapotranspiración}

En cuanto a la evapotranspiración de referencia (ETo), existe una variación interanual en los valores recogidos, con un mayor rango durante los meses de primavera-verano y menor durante el periodo de otoño-invierno.

Su determinación es más compleja que la de la precipitación ya que depende de dos procesos; la evaporación desde el suelo y desde la superficie cubierta por el cultivo, y por otro lado el fenómeno de la transpiración desde las hojas. Esta situación hace que la evapotranspiración no sólo se vea afectada por los valores climatológicos sino también por las características del suelo y los factores de la planta. En el caso del estudio, al tratarse de un solo cultivo y calcularse con datos meteorológicos para cada una de las estaciones agroclimáticas, se adoptaron valores normales. Esta hipótesis se confirma al determinar la distribución de probabilidad de los valores mensuales de evapotranspiración, que presentan una menor desviación estándar en los resultados (Figura 3). En la estimación de la reserva hídrica del suelo se utilizaron los datos medios de la ETo.

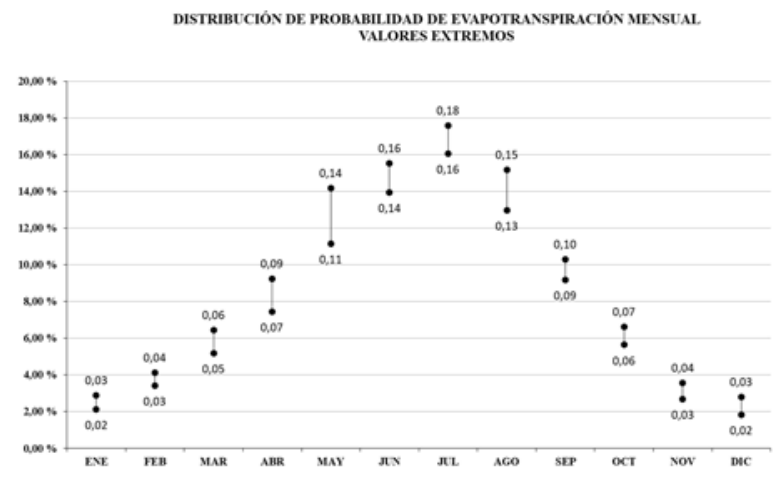

Figura 3: Distribución de probabilidad de la evapotranspiración. Valores extremos.

\subsection{Estrategia 1: Programa de riego deficitario de marzo a septiembre, regando todos los días del mes. (IDD).}

La Figura 4 muestra el patrón de reserva de agua del suelo considerando tres escenarios diferentes de precipitación y tres números diferentes de sectores. En los años lluviosos (Figura 4a), la reserva de agua se agotó de julio a agosto en la estrategia de 2 sectores, y de julio a septiembre en las estrategias de 3 sectores. No aparece estrés hídrico en la estrategia de 1 sector. La cantidad de agua aplicada también varió entre estas estrategias (Tabla 2): Para tres sectores se utilizó 161,2 mm, mientras que para un sector consumió 305,09 mm. La estrategia de 2 sectores presentó una cantidad intermedia con $212 \mathrm{~mm}$. El tiempo de riego también varió, pero en sentido contrario, desde 3182 horas al año (3 sectores) hasta 2013 horas al año en 1 sector, con un valor intermedio de 2790 horas de riego en 2 sectores.

Para la situación de precipitaciones medias (Figura $4 \mathrm{~b}$ ) no hay ningún periodo de estrés hídrico cuando se riega 1 sector. En los riegos de 2 y 3 sectores, el agotamiento de la reserva de agua del suelo se produce en la misma época (julio), aunque en el primer caso dura dos meses y en el segundo se prolonga hasta el final de la campaña de riego. En cuanto a la cantidad de agua aplicada, los resultados para 1 sector fueron de $397,3 \mathrm{~mm}$, para 2 sectores de $316,7 \mathrm{~mm}$ y para 3 sectores de $217,5 \mathrm{~mm}$. En cuanto al tiempo de riego, el resultado es mayor a medida que aumenta el número de sectores, de modo que para 1 sector fue de 2614 horas, para 2 sectores de 4168 horas y para 3 sectores de 4293 horas 


\section{Congreso Nacional de Riegos CARTAGENA 2021}
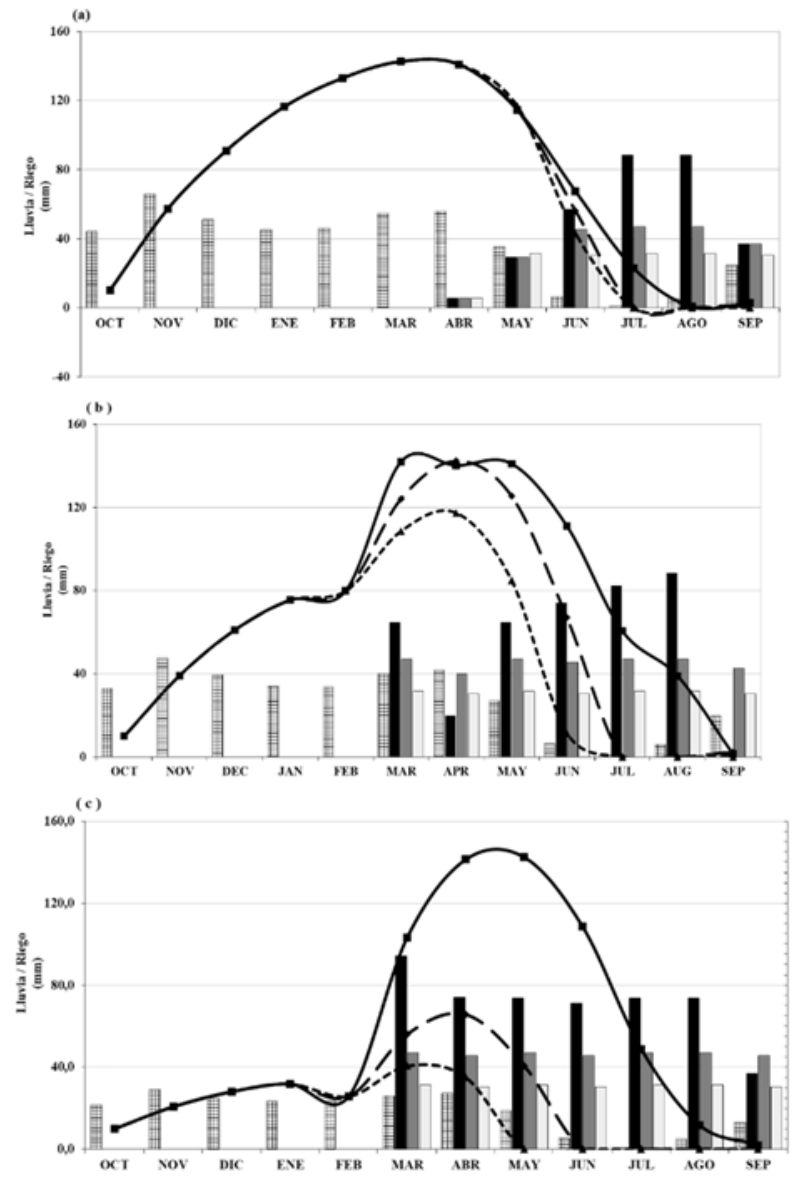

Figura 4: Patrón de la reserva de agua del suelo en el riego diario en condiciones de lluvia máxima (a), media (b) y mínima (c). Línea sólida, estrategia de 1 sector; línea de guiones largos, estrategia de 2 sectores; línea de guiones, estrategia de 3 sectores. Barras con patrón cuadrado, precipitación efectiva; barras sólidas, riego en la estrategia de 1 sector; barras claras, riego en la estrategia de 2 sectores; barras blancas, riego en la estrategia de 3 sectores.

Por último, en el supuesto más limitante (Figura 4c), el escenario de precipitaciones mínimas, no se estimó ningún período de estrés hídrico para 1 sector, pero en los demás estos períodos aumentaron. La estrategia de 2 sectores presentaría un periodo de estrés hídrico de junio a septiembre, mientras que la estrategia de 3 sectores sería aún mayor con un agotamiento del agua del suelo de mayo a septiembre. El consumo de agua aumentaría claramente (Tabla 2): en la estrategia de 1 sector sería de 497,5 mm, en la de 2 sectores de 325,3 $\mathrm{mm}$ y en la de 3 sectores de 217,5 $\mathrm{mm}$. El tiempo de riego estacional también aumentaría en todas las estrategias con 3273 horas en 1 sector y más tiempo en 2 sectores (4280 horas) y 3 sectores (4293 horas).

\subsection{Estrategia 2: Programa de riego deficitario de marzo a septiembre, riego en 20 días del mes. (ID20).}

El escenario de precipitaciones máximas presentó un periodo de estrés hídrico en todas las estrategias sectoriales. En la estrategia de 1 sector, el estrés hídrico se estimó sólo en agosto. Pero en las estrategias de los sectores 2 y 3, estos periodos fueron más amplios en los dos meses de julio a septiembre. El agua aplicada pasa de 262,2 $\mathrm{mm}$ en 1 sector a 107,4 $\mathrm{mm}$ en 3 sectores, lo que supone una gran reducción, superior al 50\%. (Tabla 2). El tiempo de riego fue máximo en la estrategia de 3 sectores con 


\section{Congreso Nacional de Riegos CARTAGENA 2021}

2119 horas por temporada y mínimo en 1 sector (1725 horas). La estrategia de 2 sectores fue intermedia pero cercana al valor máximo con 2075 horas.

El escenario de precipitaciones medias presentó los mismos resultados que la temporada de lluvias sobre el periodo de estrés hídrico. Sin embargo, la reducción de las precipitaciones de la estación media en comparación con la lluviosa aumentó el agua aplicada en torno al 40\% (370,5 mm en 1 sector, 210,9 $\mathrm{mm}$ en 2 sectores y 142,3 $\mathrm{mm}$ en 3 sectores) (Tabla 2). El aumento del tiempo de riego fue menor entre la estación de lluvias y la de precipitaciones medias (alrededor del 30\%), con un máximo en 3 sectores de 2809 horas y un mínimo en 1 sector de 2438 horas (en 2 sectores se estimó en 2775 horas).

Por último, el escenario de precipitaciones mínimas cambió claramente los resultados en comparación con los dos anteriores. La estrategia de todos los sectores presentó periodos de estrés hídrico, el sector 1 sólo de julio a agosto, pero los sectores 2 y 3 de mayo a septiembre. Esta disminución de las precipitaciones aumentaría el consumo de agua en todos los sectores de la estrategia hasta los valores máximos en 1 sector de la estrategia $(412,3 \mathrm{~mm})$ y el mínimo de 3 sectores $(142,3 \mathrm{~mm})$, con un valor intermedio en 2 sectores $(212,8 \mathrm{~mm})$.

\subsection{Programa de riego deficitario de marzo a septiembre con riego controlado en agosto. (RDI).}

El escenario de precipitaciones máximas, la estrategia de todos los sectores presentó un periodo de estrés hídrico. En 1 sector, el estrés hídrico se estimó sólo en agosto, pero en los otros dos el período fue más amplio, de julio a septiembre en 3 sectores, pero de julio a agosto en 2 sectores. En estas condiciones, el agua aplicada fue máxima en 1 sector con 284,1 mm y mínima en 3 sectores $129,7 \mathrm{~mm}$, con un valor intermedio en 2 sectores con 182,6 mm (Tabla 2). El tiempo de riego se estimó en 1869 horas (1 sector), 2403 horas (2 Sectores) y 2560 (3 Sectores).

El escenario de precipitaciones medias el periodo de estrés es más largo en 3 sectores (tres meses), que en 2 sectores ( 2 meses), y en 1 sector ( 1 mes), aunque en 1 sector se produce durante el mes de agosto, mientras que en las estrategias de 2 y 3 sectores comienza en julio. A medida que aumentamos el número de sectores tenemos una disminución del agua aplicada y un aumento del tiempo de riego. La estrategia del sector 1 presentó un agua aplicada de $377,8 \mathrm{~mm}$, mientras que los sectores 2 y 3 fueron de $266,8 \mathrm{~mm}$ y $186 \mathrm{~mm}$, respectivamente. Por otro lado, el tiempo de riego varió entre 2485 horas (para 1 sector) y 3671 horas (para 3 sectores), presentando un valor intermedio para 2 sectores de 3510 horas.

Por último, en condiciones de lluvia mínima (Figura 9c), los periodos de estrés hídrico aumentaron en todas las estrategias. Fueron de junio a septiembre en 2 sectores y de mayo a septiembre en 3 sectores. Sólo en la estrategia de 1 sector, el periodo de estrés hídrico no aumentó y se estimó únicamente durante el mes de agosto. El agua aplicada aumentó claramente con 472,9 mm en 1 sector, 278,2 mm en 2 sectores y $186 \mathrm{~mm}$ en 3 sectores. Este nivel de agua aplicada produjo un aumento del tiempo de riego de 3111 horas en 1 sector, 3660 horas en 2 sectores y 3671 horas en 3 sectores.

\section{4.- Discusión.}

Los resultados de los diferentes escenarios presentaron una amplia gama de consumos de agua y periodos de estrés hídrico que podrían afectar al beneficio de las explotaciones olivareras. Los agricultores en la mayoría de los escenarios considerados manejarían una programación de riego deficitaria debido a las grandes limitaciones de disponibilidad de agua, en tiempo y cantidad. La selección de una estrategia de riego tiene que considerar, principalmente, dos factores: cuándo se produciría este periodo de estrés hídrico y la cantidad de agua aplicada. Los resultados esperados en cuanto a rendimiento cambiarían en gran medida según el momento de este periodo de estrés hídrico. El estrés hídrico más temprano se estimó en mayo para el escenario de precipitaciones mínimas en la estrategia de 3 


\section{Congreso Nacional de Riegos CARTAGENA 2021}

sectores en todas las programaciones de riego y en la estrategia de 2 sectores cuando se modeló la programación ID20. El mes de mayo es la fecha habitual de plena floración en esta zona. Hay varios trabajos que han descrito este periodo fenológico como el más sensible a la sequía [7-35]). Aunque estas condiciones son muy poco frecuentes en la cuenca mediterránea, y por ello el olivo es un cultivo frutal de secano, los cambios climáticos podrían aumentar el número de temporadas en las que esto ocurra [36]). Por lo tanto, la estrategia de 3 sectores era la menos sostenible, ya que los recursos hídricos se desperdiciarían en los escenarios de precipitaciones más limitantes e incluso se esperarían diferencias bajas con las condiciones de secano.

Varios de los escenarios considerados podrían retrasar el periodo de estrés hídrico a después de la plena floración y así escapar de la mayor reducción del rendimiento. Este es el caso de las condiciones de lluvia mínima en la estrategia de 2 sectores con riego diario y programación de RDI. El periodo de endurecimiento del hueso se considera el periodo más resistente a la sequía en el olivo [37]. Es común sugerir 49-56 días después de la floración completa como la fecha más probable para el inicio de este período [38], que, para el área de estudio, podría estimarse que comienza alrededor de principios de julio. Luego, en junio, es probable que el estrés hídrico se produzca durante el crecimiento del endocarpio. En esta etapa fenológica se ha estimado una reducción del rendimiento en torno al $20 \%$ en un nivel de estrés hídrico moderado [32-39]). Pero, un nivel de estrés hídrico severo podría incluso afectar al rendimiento de la siguiente temporada [40]. Entonces, estas estrategias podrían ser adecuadas si garantizan una gran reducción del agua aplicada y el olivicultor podría asumir esta importante reducción del rendimiento. El periodo de estrés hídrico por endurecimiento del hueso, probablemente alrededor de julio en esta zona, reduciría el efecto sobre el rendimiento. El nivel de estrés hídrico fuerte a mediados de verano no afectó significativamente al rendimiento en varios olivares de riego, pero las tendencias fueron similares a la anterior con una reducción del rendimiento del 20\% (7-41). Sin embargo, estos resultados se obtuvieron en olivares más densos y se esperaría un menor nivel de estrés hídrico en el tradicional. En condiciones de estrés hídrico moderado la reducción del rendimiento sería casi nula, más aún si se esperan lluvias otoñales antes de la cosecha [7-41]. Además, la acumulación de aceite podría no verse afectada con el estrés hídrico durante este periodo [42]). Entonces, todas las programaciones de riego en el escenario de 1 sector serían adecuadas y también las de 2 sectores si no se dan condiciones de lluvia mínima. Esta discusión se centra en las aceitunas destinadas a la almazara ya que se recuperan con las lluvias de otoño, pero no en las aceitunas de mesa ya que el tamaño del fruto es muy sensible al estrés hídrico y es muy importante en este tipo de producción [43].

La disponibilidad de agua cambia fuertemente entre las plantaciones de olivos e, incluso, entre las estaciones, ya que se puede aplicar una restricción severa durante los períodos de sequía. En España, la cantidad máxima de riego estacional es limitante y una disponibilidad común de agua en los huertos de olivos es de $150 \mathrm{~mm}$ [3]. Esta agua aplicada es mucho menor que la estimada en todas las estrategias de los sectores 1 y 2 (Tabla 2). Este resultado sugiere que la sostenibilidad del riego en la explotación olivarera tradicional es muy limitante si no se consideran programaciones de riego precisas adicionales. En estas condiciones de muy baja disponibilidad de agua, el riesgo de no obtener mejoras en comparación con el olivar sin riego sería muy posible. Moriana y otros autores, [8] (2007) no encontraron diferencias significativas en un olivar tradicional de Cornicabra a una distancia de $12 * 12 \mathrm{~m}$ con cantidades de agua más restrictivas que en el presente trabajo $(75 \mathrm{~mm})$ y alcanzando niveles de estrés hídrico signficativamente mayores que el riego con el $100 \%$ de ETc durante el endurecimiento del hueso. Entonces, esta cantidad de agua podría utilizarse para asegurar un estado hídrico óptimo o casi óptimo en la etapa fenológica más limitante (es decir, la plena floración) y no para un riego deficitario sostenible. En tales condiciones, la determinación precisa del estado del agua podría ser muy importante [44]). 


\section{Congreso Nacional de Riegos CARTAGENA 2021}

Tabla 2: Resumen del agua aplicada $(\mathrm{mm})$, de la reserva de agua $(\mathrm{mm})$ y del tiempo de riego estacional (horas) en las tres condiciones de precipitación (estación lluviosa, media y seca), con los tres calendarios de riego (diario, 20 días al mes y sin riego en agosto (RDI)) en la estrategia de los 3 sectores.

\begin{tabular}{|l|l|l|l|l|l|l|l|l|l|}
\cline { 2 - 9 } \multicolumn{2}{c|}{} & \multicolumn{3}{c|}{ S1: IDD } & \multicolumn{3}{c|}{ S2:ID20 } & \multicolumn{3}{c|}{ S3:RDI } \\
\cline { 2 - 10 } & $\mathbf{1}$ sector & 2 sector & $\mathbf{3}$ sector & 1 sector & 2 sector & 3 sector & 1 sector & 2 sector & 3 sector \\
\hline $\begin{array}{l}\text { Agua aplicada (mm) } \\
\text { con máximas lluvias }\end{array}$ & 305,9 & 212,0 & 161,2 & 262,2 & 157,7 & 107,4 & 284,1 & 182,6 & 129,7 \\
\hline $\begin{array}{l}\text { Tiempo de riego con } \\
\text { lluvias máximas (h) }\end{array}$ & 2013 & 2790 & 3182 & 1725 & 2075 & 2119 & 1869 & 2403 & 2560 \\
\hline $\begin{array}{l}\text { Reserva de agua (mm) } \\
\text { con lluvias máximas }\end{array}$ & 899,1 & 863,7 & 850,6 & 902,7 & 848,3 & 818,1 & 1051,8 & 899,1 & 850,6 \\
\hline $\begin{array}{l}\text { Agua aplicada (mm) } \\
\text { con lluvias medias }\end{array}$ & 397,3 & 316,7 & 217,5 & 370,5 & 210,9 & 142,3 & 377,7 & 266,8 & 186,0 \\
\hline $\begin{array}{l}\text { Tiempo de riego con } \\
\text { lluvias medias (horas) }\end{array}$ & 2614 & 4168 & 4293 & 2438 & 2775 & 2809 & 2485 & 3510 & 3671 \\
\hline $\begin{array}{l}\text { Reserva de agua (mm) } \\
\text { con lluvias medias }\end{array}$ & 900,1 & 727,0 & 587,6 & 800,1 & 580,5 & 511,1 & 908,2 & 718,4 & 587,6 \\
\hline $\begin{array}{l}\text { Agua aplicada (mm) } \\
\text { con mínimas lluvias }\end{array}$ & 497,5 & 325,3 & 217,5 & 412,3 & 212,8 & 142,3 & 472,9 & 278,2 & 186,0 \\
\hline $\begin{array}{l}\text { Tiempo de riego en con } \\
\text { lluvias mínimas (h) }\end{array}$ & 3273 & 4280 & 4293 & 2713 & 2800 & 2809 & 3111 & 3660 & 3671 \\
\hline $\begin{array}{l}\text { Reserva de agua (mm) } \\
\text { con lluvias mínimas }\end{array}$ & 674,6 & 279,0 & 191,9 & 403,9 & 189,6 & 159,4 & 715,6 & 279,0 & 191,9 \\
\hline
\end{tabular}

\section{5.- Conclusiones.}

La sostenibilidad del riego en los olivares tradicionales debe tener en cuenta varios aspectos para asegurar una gestión eficiente del agua. La conservación de los recursos hídricos es el más importante, pero también debe considerarse el uso de estos recursos naturales para mejorar los beneficios de los olivicultores. El trabajo actual demuestra que la estrategia de riego más común en una de las zonas tradicionales de producción de aceitunas más importantes del mundo no es adecuada. La estrategia de los 3 sectores presentó el periodo más largo de estrés hídrico y, lo más importante, en las etapas fenológicas más críticas cuando se produjo el escenario de lluvias mínimas. Entonces, adaptar el agua actual disponible a un riego deficitario sostenible que, en el escenario de menor pluviometría, incluso reduce fuertemente el rendimiento, es un desperdicio de agua y de dinero del agricultor. Esto supone que los olivares tradicionales tienen que cambiar a estrategias de 1 ó 2 sectores con programación diaria o RDI. Sin embargo, estas estrategias suponen un gran aumento del agua aplicada que podría no ser sostenible, principalmente en el escenario de precipitaciones mínimas. En estas condiciones, las estrategias de 1 ó 2 sectores podrían considerar una programación de riego precisa y concentrada que al menos asegure unas condiciones cercanas a las óptimas en las etapas fenológicas más sensibles.

\section{Referencias}




\section{Congreso Nacional de Riegos CARTAGENA 2021}

1. FAOSTAT, Datos sobre Alimentación y Agricultura, 2020. Organización de las Naciones Unidas para la Alimentación y la Agricultura. http://www.fao.org/faostat/es/\#data/QC/visualize.

2. MAPA, Ministerio de Agricultura, Pesca y Alimentación, 2018. “Anuarios de Estadística Agraria”. Parte Tercera. Capitulo 07.07-12. https://www.mapa.gob.es/es/estadistica/temas/publicaciones/anuario-de-estadistica/default.aspx.

3. C.H.G., Confederación Hidrográfica del Guadalquivir, 2019. “Datos y capas de riegos en la provincia de Jaén”. https://www.chguadalquivir.es/inicio.

4. Khan S,, Tariq R,, Yuanlai C., and Blackwell J., 2004. “Can irrigation be sustainable?” En: New Directions for a Diverse Planet. Proceedings of the 4th International Crop Science Congress. Brisbane, Australia. www.cropscience.org.au

5. Schoonhoven, Y., and Runhaar, H., 2018. "Conditions for the adoption of agro-ecological farming practices: a holistic framework illustrated with the case of almond farming in Andalusia". International Journal of Agricultural Sustainability 16(3):1-13. DOI:10.1080/14735903.2018.1537664.

6. Lorite, I.J.; Gabaldón-Leal, C.; Ruiz-Ramos, M.; Belaj, A.; de la Rosa, R.; León, L.; Santos, C. , 2018. “Evaluation of olive response and adaptation strategies to climate change under semi-arid conditions". Agricultural Water Management 247261. DOI: https://doi.org/10.1016/j.agwat.2018.04.008.

7. Moriana, A., Orgaz, F., Pastor, M., and Fereres, E., 2003. "Yield Responses of a Mature Olive Orchard to Water Deficits". Journal of the American Society for Horticultural Science. 128(3): pp, 425-431. DOI:10.21273/JASHS.128.3.0425.

8. Moriana, A., Gomez, A., Desamparados, S., Perez, D., Olmedilla, N, Ribas, F., and Fregapane, G., 2007. "Influence of different irrigation strategies in a traditional Cornicabra cv. olive orchard on virgin olive oil composition and quality". Food Chemistry Vol 100, Issue 2:568-578. DOI: https://doi.org/10.1016/j.foodchem.2005.09.075.

9. Giron, I., Martín, M.J., Corell, M., et al, 2016. “Umbrales Sostenibles de Estrés Hídrico durante el Endurecimiento del Hueso en la Producción de Aceituna de Mesa". Conference Paper at XXXIV Congreso Nacional de Riegos. Sevilla.

10. Diaz, A., Fernandez, J.E., Perez, A., et al., 2018. The olive tree under water stress: fitting the pieces of response mechanisms in the crop performance puzzle. Water Scarcity and Sustainable Agriculture in Semiarid Environment. Tools, Strategies, and Challenges for Woody Crops. Academic Press. pp. 439-479. ISBN: 978-0-12-813164-0

11. MAPA, Ministerio de Agricultura, Pesca y Alimentación, 2019. “Encuesta sobre Superficies y Rendimientos Cultivos. Resultados 2019", ESYRCE. Apartado 3.3. N.I.P.O.: 003-19-051-9. https://www.mapa.gob.es/es/estadistica/temas/estadisticas-agrarias/boletin2019 tcm30-536911.pdf/.

12. Penco, J.M., 2020. “Aproximacion a los costes del cultivo del olivo". Desarrollo y conclusiones del estudio AEMO. Spanish Association of Olive Municipalities. https:/www.aemo.es/slides/slide/estudio-de-costes-aemo-2020-241. Last access: 08/04/2021.

13. CAP, 2015. "Plan Director del Olivar". Consejería de Agricultura, Ganadería, Pesca y Desarrollo Sostenible. Decreto 103/2015, de 10 de marzo. BOJA № 54, (19/03/2015).

14. Rojas, R., Roldán, J., López, R., Alcaide, M., and Camacho, E, 1996. “El riego del olivar en la provincia de Jaén (II): programación de riegos". Ingeniería del Agua. 3(1). DOI:10.4995/ia.1996.2691.

15. Pastor, M., Orgaz, F., Vega, V., Hidalgo, J., Castro, J., Nieto, J., Aguilar, J. Fernandez, E., Morales, J., and Soria, L., 1998. "Programación del riego y la fertilización en olivares de la provincia de Jaén". Junta de Andalucia. Consejería de Agricultura y Pesca de la Junta de Andalucía. ISBN: 84-89802-30-0.

16. Granados, A., 2013. "Criteria for dimensioning irrigation networks that are robust to changes in crop alternatives". Doctoral thesis. ETSI Caminos, Canales y Puertos. Universidad Politecnica de Madrid. Reg_ 16101. DC: http://oa.upm.es/16101/. OAI: oai:oa.upm.es:16101.

17. Alduan, A., and Monserrat, J., 2009. “Estudio comparativo entre la organización a la demanda o por turnos en redes de riego a presión". Ingeniería del agua, [S.1.], v. 16, n. 3, p. 235-242, sep. 2009. ISSN 1886-4996. DOI: https://doi.org/10.4995/ia.2009.2951.

18. Peragón, J.M., Delgado, A., Rodríguez, J.A., and Pérez, F.J., 2016. “A GIS-based decision tool for reducing salinization risks in olive orchards". Agricultural Water Management. 33-41. pp 166. DOI:10.1016/j.agwat.2015.12.005

19. R.I.A.A, 2020. Red de Información Agroclimática de Andalucía. I.F.A.P.A., (Instituto de Investigación y Formación Agraria y Pesquera). Consejería de Agricultura, Ganadería, Pesca y Desarrollo Sostenible: https://www.juntadeandalucia.es/agriculturaypesca/ifapa/riaweb/web/.

20. FAO, Organización de las Naciones Unidas para la Alimentación y la Agricultura, 2006. “Evapotranspiración del cultivo. Guías para la determinación de los requerimientos de agua de los cultivos". pp. 89134.http://www.fao.org/3/x0490s/x0490s00.htm

21. CAP, 2001. "Programación de riegos en olivar". Consejería de Agricultura, Ganadería, y Pesca. Serie: Olivicultura y Elaiotecnia. ISBN: 84-89802-33-5.

22. Andrades, M.S., and Muñez, C., 2012. "Fundamentos de climatología”. Universidad de La Rioja. Coleccion: Material didáctico. Agricultura y Alimentación. 2ª edicion. ISBN: 978-84-695-2799-3 


\section{Congreso Nacional de Riegos CARTAGENA 2021}

23. Chaves, V.M., 2006. “Manejo del riego por goteo en olivo y respuesta de la planta al regimen hídrico". Memoria del XLIII Curso Internacional de Edafología y Biología Vegetal. Departamento de Sostenibilidad del Sistema Suelo-Planta-Agua. Instituto de Recursos Naturales y Agrobiología de Sevilla (IRNASS). Consejo Superior de Investigaciones Cientificas, (CSIC).. http://hdl.handle.net/10261/134075.

24. Fereres, E., and Villalobos, F.J., 2016. "Deficit Irrigation. Principles of Agronomy for Sustainable Agriculture”. Springer International Publishing. pp. 281-294. ISBN: 9783319834450. doi:10.1007/978-3-319-46116-8 21.

25. Orgaz, F., Hidalgo, J., Castro, J.A., et al., 1999. “Respuesta del olivar tradicional a diferentes estrategias y dosis de agua de riego". Investigación agraria Producción y protección vegetales. 14 (3). pp. 393-404.

26. Allen, R.G., Pereira, L.S., Raes, D., and Smith, M., 1998. “Crop evapotranspiration. Guidelines for computing crop water requirements". FAO Irrigation and drainage paper 56. Food and Agriculture Organization, Rome.

27. Castel, J.R., and Fereres, E., 1982. "Responses of Young Almond Trees to Two Drought Periods in the Field". Journal of Horticultural Science. 57(2). pp. 175-187. DOI:10.1080/00221589.1982.11515038.

28. Aguilera, F.,and Ruiz, L., 2009. “Periodo de floración del olivo (Olea europaea L.) en la provincia de Jaén”. Departamento de Biología Animal, Biología Vegetal y Ecología. Universidad de Jaén. Conference presented at Expoliva.

29. Larios, J.M., 2008. "Respuesta del olivo a tratamientos con riego deficitario". Instituto de Recursos Naturales y Agrobiologia de Sevilla, (IRNASS). Consejo Superior de Investigaciones Cientificas, CSIC. Doctoral Thesis. http://hdl.handle.net/10261/139518.

30. Lopez, G., Arbones, A., Girona, J., and Marsal, J., 2008. “Response of peach trees to regulated deficit irrigation during stage II of fruit development and summer pruning". Spanish Journal of Agricultural Research. DOI: 10.5424/sjar/2008063-340.

31. Hidalgo, J.J., and Vega, V., 2008. “Estrategias de riego deficitario controlado en un olivar de aceituna de mesa cv Manzanilla. Resultados de los 3 primeros años de un ensayo de larga duración". Conference Paper at XXVI Congreso Nacional de Riegos; AERYD, Asociación Española de Riegos y Drenajes: Huesca, 24-26 de junio. pp. 31-32.

32. Gómez del Campo, M., 2013. "Summer deficit irrigation in a hedgerow olive orchard cv. Arbequina: relationship between soil and tree water status, and growth and yield components". Spanish Journal of Agricultural Research. 11(2). pp. 547-557. http://dx.doi.org/10.5424/sjar/2013112-3360.

33. Lavee, S., Hanoch, E., Wodner, M., and Abramowitch, H., 2007. "The effect of predetermined deficit irrigation on the performance of cv. Muhasan olives (Olea europaea L.) in the eastern coastal plain of Israel". Scientia Horticulturae, 2007. (112). pp. 156-163. doi:10.1016/j.scienta.2006.12.017.

34. D'Amato, R., Proietti, P., Onofri, A., et al., 2017. “Biofortification (Se): Does it increase the content of phenolic compounds in virgin olive oil (VOO)?". Plos One. 12(4):e0176580. doi:10.1371/journal.pone.0176580

35. Rapoport, HF., Hammami, SBM., Martins, P., Perez-Priego, O., and Orgaz, F., 2012. "Influence of water deficits at different times during olive tree inflorescence and flower development". Environmenatl and Experimental Botany 77:227-233

36. López Bernal, A., Testi, L., and Villalobos, F.J., 2019. “Olivar y cambio climático, predicciones de un modelo de simulación". Vida rural, ISSN 1133-8938, № 470, págs. 38-42.

37. Goldhamer, D.A., 1999. “Regulated Deficit Irrigation for California Canning Olives”. Acta Horticulturae.(474). pp.369372. DOI:10.17660/ActaHortic.1999.474.76.

38. Hammami, S., Manrique, T., and Rapoport, H., 2011. “Cultivar-based fruit size in olive depends on different tissue and cellular processes throughout growth". Scientia Horticulturae. (130). pp. 445-451. DOI:10.1016/j.scienta.2011.07.018.

39. Gucci, R., Lodolini, E., and Rapoport, HF., 2007. "Productivity of olive trees with different water status and crop load". Journal of Horticultural Science \& Biotechnology 82, 648-656.

40. Gucci, R., Caruso, G., Gennai, C., Esposto, S., Urbani, S., and Servili, M., 2019. "Fruit growth, yield and oil quality changes induced by deficit irrigation at different stages of olive fruit development". Agricultural Water Management 212, 88-98.

41. Ahumada, L., Ortega, S., Searles, P., and Retamales, J., 2017. "Yield and Water Productivity Responses to Irrigation Cutoff Strategies after Fruit Set Using Stem Water Potential Thresholds in a Super-High Density Olive Orchard". Frontiers Research Foundation. Frontier in Plant Science. pp. 8-11. DOI:10.3389/fpls.2017.01280.

42. López-Bernal, A., Fernandes-Silva, A.A., Vega, V.A., Hidalgo, J.C., León, L., Testi, L., and Villalobos, FJ., 2021. “A fruit growth approach to estimate oil content in olives". Europ. J. Agron. 123. DOI: 0.1016/j.eja.2020.126206

43. Girón, I.F., Corell, M., Galindo, A., Torrecillas, E., Morales, D., Dell'Amico, J., Torrecillas, A., Moreno, F., and Moriana, A., 2015. "Changes in the physiological response between leaves and fruits during a moderate water stress in table olive trees". Agricultural Water Management 148:280-286

Ben-Gal, A., Ron, Y., Yermiyahu, U., Zipori, I., Naoum, S., and Dag, A., 2021. "Evaluation of regulated deficit irrigation strategies for oil olives: A case study for two modern Israeli cultivars". Agric. Water Manage. 245, doi 10.1016/j.agwat.2020.106577. 